SEX WORK

\title{
Acceptability and feasibility of continuous diaphragm use among sex workers in Madagascar
}

\author{
F Behets, A Norris Turner, K Van Damme, N L Rabenja, N Ravelomanana, K Zeller, \\ J R Rasolofomanana
}

Sex Transm Infect 2005;81:472-476. doi: 10.1136/sti.2005.015107

See end of article for authors' affiliations

\section{Correspondence to:}

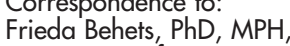
Department of Epidemiology, University of North Carolina at Chapel Hill, 2102-D McGavran-Greenberg

Hall, CB \#7435, Chapel Hill, NC 27599-7435, USA; frieda_behets@unc. edu

Accepted for publication 9 April 2005

Objectives: The diaphragm, a woman controlled, reusable contraceptive device, might prevent some sexually transmitted infections (STIs). We assessed the acceptability and feasibility of use of silicone WideSeal Arcing Diaphragms (Milex Products, Chicago, IL, USA) by sex workers in Madagascar.

Methods: Over 8 weeks, we evaluated method acceptability by examining patterns of and problems with women's diaphragm use. We also evaluated several measures of study feasibility, including recruitment and follow up methods.

Results: 91 women from three cities (Antananarivo, Tamatave, and Mahajanga) participated, and 87 (96\%) completed follow up. At enrolment, participants reported a median of six sex acts with five clients in the previous week. During the follow up period, participants reported a median of three sex acts with three clients during the previous 2 days, and self reported continuous diaphragm use during the previous day increased from $87 \%$ to $93 \%$. Seven women became pregnant (incidence 53 pregnancies per 100 woman years). Self reported use of male condoms and diaphragms was fairly constant over the study period: women reported condom use in $61 \%$ to $70 \%$ of acts and diaphragms in $95 \%$ to $97 \%$ of acts. The number of participants reporting diaphragm problems decreased from $15(16 \%)$ at the first visit to six $(7 \%)$ at the final visit. 20 women $(22 \%)$ needed replacement devices during follow up because their original diaphragms were lost, were the wrong size, or became seriously damaged.

Conclusions: Given the high use and steady decrease in reported problems during the study, we believe diaphragms are acceptable and feasible in this resource poor, low education sex worker population.

B ecause many women lack the social and economic power to insist that their sex partners remain monogamous or use condoms, millions remain vulnerable to sexually transmitted infections (STIs). For years researchers have discussed the need for women controlled STI prevention options that could be used without men's permission or even knowledge. ${ }^{1}$ The diaphragm, a reusable contraceptive device approved worldwide for prevention of pregnancy, ${ }^{2}$ has attracted renewed attention as a means to control STIs and HIV..$^{3-5}$ By covering the cervix during exposure to gonorrhoea, chlamydia, or HIV (and possibly other STIs), diaphragms may block these pathogens from reaching their preferred infection site. Diaphragms have a proved safety record because of their long history as birth control, and they may also be used clandestinely when partners reject male condoms. While no yet published randomised trials have evaluated the effectiveness of the diaphragm against STI acquisition, several observational studies report a protective effect of diaphragm use against gonococcal and chlamydial infection and their long term sequelae..$^{6-10}$

This study was conducted in Madagascar, a country with both high STI rates and ample evidence that current strategies for STI prevention are inadequate. In 1997, among women who reported not trading sex for money who presented with genitourinary symptoms in the capital Antananarivo, the prevalence of gonorrhoea and chlamydia was $9.7 \%$ and $8.9 \%$, respectively. ${ }^{11}$ In $2001,75-78 \%$ of asymptomatic female sex workers in Madagascar had at least one curable STI, and up to $30 \%$ had gonorrhoea or chlamydia. ${ }^{12}$ Rates remained high even after presumptive treatment and condom promotion. ${ }^{12}$ Development of new STI control methods is a crucial research priority, both to directly reduce the STI burden and because STIs are likely to increase risk of HIV acquisition. ${ }^{13-15}$
We plan to conduct a randomised trial to evaluate the effectiveness of the diaphragm against Chlamydia trachomatis and Neisseria gonorrhoeae in female sex workers in Madagascar, and we completed the 8 week pilot study described here to assess the acceptability and feasibility of diaphragm use in this population. In addition to measuring changes in diaphragm and condom use patterns, problems with study products, and reasons for discontinuation, we also assessed several study design components such as recruitment and follow up methods. Formative research on acceptability and feasibility was particularly appropriate because diaphragms are virtually unknown in Madagascar.

\section{METHODS}

We recruited women who self identified as sex workers in three urban centres in Madagascar (Antananarivo, Tamatave, and Mahajanga). National STI treatment guidelines in Madagascar recommend that all sex workers be seen quarterly at government health clinics for routine STI testing and treatment, although compliance is voluntary and no formal sex worker registry exists. Peer counsellors recruited participants from clinics when they presented for their STI visits. Eligible women were inconsistent condom users (reporting less than $100 \%$ condom use with clients in the past month), not pregnant or desiring pregnancy in the next 6 months, with healthy vagina and cervix upon visual inspection, and able to be fitted with a diaphragm. We followed women for 8 weeks.

Women provided written informed consent before screening, and eligible participants signed a second consent form at enrolment. The institutional review board at the University of

Abbreviations: EC, emergency contraception; RPR, rapid plasma reagin; STI, sexually transmitted infections; UTI, urinary tract infection 
Table 1 Demographic and reproductive characteristics of study participants at enrolment

\begin{tabular}{|c|c|c|c|c|c|c|c|c|}
\hline \multirow[b]{2}{*}{ Characteristic } & \multicolumn{2}{|c|}{ Antananarivo $(n=30)$} & \multicolumn{2}{|c|}{ Tamatave $(n=31)$} & \multicolumn{2}{|c|}{ Mahajanga $(n=30)$} & \multicolumn{2}{|c|}{ Total $(n=91)$} \\
\hline & Median & (range) & Median & (range) & Median & (range) & Median & (range) \\
\hline Age & 27 & $(18-42)$ & 25 & $(20-45)$ & 29.5 & $(18-48)$ & 28 & $(18-48)$ \\
\hline Number of pregnancies & 2.5 & $(0-7)$ & 2 & $(0-6)$ & 2 & $(0-5)$ & 2 & $(0-7)$ \\
\hline Completed years of schooling & 4 & $(0-8)$ & 5 & $(0-11)$ & 5 & $(0-13)$ & 5 & $(0-13)$ \\
\hline Number of sex acts, last week & 11 & $(0-27)$ & 4 & $(0-32)$ & 7 & $(0-40)$ & 6 & $(0-40)$ \\
\hline Number of sex partners, last week & 10 & $(0-26)$ & 3 & $(0-32)$ & 6 & $(0-40)$ & 5 & $(0-40)$ \\
\hline \multirow[t]{2}{*}{ Number of new sex partners, last week } & 1 & $(0-13)$ & 3 & $(0-32)$ & 3 & $(0-34)$ & 3 & $(0-34)$ \\
\hline & No & (\%) & No & $(\%)$ & No & $(\%)$ & No & $(\%)$ \\
\hline \multicolumn{9}{|l|}{ Occupation } \\
\hline None & 20 & (67) & 13 & $(42)$ & 24 & $(80)$ & 57 & (63) \\
\hline Trade/commerce & 3 & (10) & 8 & $(26)$ & 3 & (10) & 14 & (15) \\
\hline Professional & 0 & (0) & 1 & (3) & 0 & (0) & 1 & (1) \\
\hline Domestic & 0 & (0) & 9 & (29) & 1 & (3) & 10 & (11) \\
\hline Other & 7 & (23) & 0 & (0) & 2 & (7) & 9 & (10) \\
\hline \multicolumn{9}{|l|}{ Current contraceptive method } \\
\hline None & 18 & $(60)$ & 23 & (74) & 7 & (23) & 48 & (53) \\
\hline Oral & 1 & (3) & 6 & (19) & 3 & (10) & 10 & (11) \\
\hline Injectable & 4 & (13) & 2 & (6) & 6 & (20) & 12 & (13) \\
\hline Condom & 7 & (23) & 0 & (0) & 13 & (43) & 20 & (22) \\
\hline Other & 0 & (0) & 0 & (0) & 1 & (3) & 1 & (1) \\
\hline Used condom at last vaginal sex & 22 & (73) & 23 & (74) & 13 & (43) & 58 & (64) \\
\hline Ever genital ulcer & 9 & (30) & 11 & (35) & 14 & (47) & 34 & (37) \\
\hline Ever oral sex & 5 & (17) & 3 & (10) & 1 & (3) & 9 & (10) \\
\hline Ever anal sex & 2 & (7) & 1 & (3) & 0 & (0) & 3 & (3) \\
\hline
\end{tabular}

North Carolina at Chapel Hill and the Comité d'Ethique auprès du Ministère de la Santé in Madagascar approved the study.

At screening, participants received pelvic examinations to assess clinical signs of vaginitis and cervicitis, urinalysis (to evaluate leucocytes in urine) to screen for urinary tract infection (UTI) (Multistix 10 SG Reagent Strips for Urinalysis, Bayer Corporation, IN, USA), urine dipstick for pregnancy testing (Clearview hCG Combo, Wampole Laboratories, NJ, USA), and blood testing for syphilis (BD Macro-Vue RPR Card Tests, Becton, Dickinson and Company, MD, USA). All women with symptoms suggestive of UTI and positive urine test received UTI treatment. Women with genital ulcers were treated according to Madagascar's national guidelines. ${ }^{12}$

Women deemed eligible at screening were enrolled immediately. Staff administered structured questionnaires capturing participants' health and behavioural characteristics, sexual history, contraception, and condom use. We dispensed presumptive treatment for gonococcal and chlamydial infections to all women (500 mg ciprofloxacin and $1 \mathrm{~g}$ azithromycin) and then 1 week later, at the first follow up visit, for bacterial vaginosis and trichomoniasis $(2 \mathrm{~g}$ metronidazole).

Study clinicians fitted each woman for a diaphragm according to standard clinical guidelines, selecting the largest

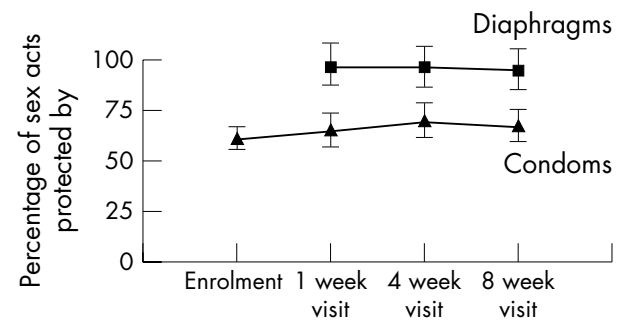

Figure 1 Percentage and 95\% confidence intervals of sex acts protected by male condoms or diaphragms at enrolment and follow up visits." (*The enrolment questionnaire asked about use of condoms over the past week; the follow up questionnaire asked about condom and diaphragm use over the past 2 days.) size that was comfortable to the participant and remained properly placed with valsalva. Clinicians verified that women could correctly insert and withdraw their diaphragms and provided extensive instructions about method use, cleaning and storage.

Women were instructed to wear the diaphragm at all times, removing it once daily for cleaning and for bathing or vaginal hygiene, then immediately replacing it ("continuous use" $^{\prime \prime}$. Lubricant gel was not provided and its use was not encouraged. We instructed women to remove the device only when at least 6 hours had passed since the last sex act, and to leave it in place for removal by clinicians on visit days. (If fewer than 6 hours had passed since the last sex act when women returned for follow up visits, clinicians did not remove the diaphragm and the examination was rescheduled). We told women that diaphragms were developed for contraception, but that they do not provide adequate pregnancy protection without spermicide. Participants were also given information about emergency contraception (EC) and told that if they had unprotected sex and wanted to reduce the risk of pregnancy, they could purchase discounted EC for $\$ 1$ from the clinic. We told women that the diaphragm was not known to protect against STIs.

At each follow up visit (1, 2, and 8 weeks after enrolment), women were interviewed to assess problems with and changes in diaphragm and condom use. To improve recall, we asked only about sexual practices over the previous 2 days. At the 1 week visit, those women who were seropositive for syphilis at enrolment were treated following the national guidelines (three doses of 2.4 MIU benzathine penicillin at 1 week intervals). ${ }^{12}$ UTI screening was performed at each visit; UTI treatment ( $100 \mathrm{mg}$ nitroxocine, three times daily for 8 days) was dispensed to symptomatic women who had leucocytes in their urine. Pregnancy testing was repeated at 4 weeks and 8 weeks.

We gave each woman a silicone Wide-Seal Arcing Diaphragm (Milex Products, Chicago, IL, USA), and as many male condoms as they wanted (spermicide free Protector Plus latex condoms). All women received safer sex counselling and were instructed to use condoms with every sex act.

Using the total number of reported sex acts as the offset variable, Poisson regression models were run to generate the 
proportion of sex acts protected by condoms and diaphragms at each study visit. We analysed the data using SAS (SAS Institute, Version 9.1, Cary, NC, USA).

\section{RESULTS}

The study took place from April through July 2004. All women $(n=97)$ approached by study staff agreed to be screened (100\% response rate). Ninety one women met the inclusion criteria and were enrolled: 30 in Antananarivo, 31 in Tamatave, and 30 in Mahajanga; 87 participants (96\%) were followed for the 8 week study duration.

Table 1 describes baseline demographic characteristics of enrolled women. Participants' median age was 28 years (range 18-48). They had completed a median of 5 years of education. The majority (84\%) were unmarried and not living with partners. Most (63\%) reported no occupation, though some were domestic workers $(11 \%)$, and others worked in trade (15\%). More than half (53\%) reported no contraceptive method, while 13\% used injectables and another $11 \%$ took oral contraceptives. Twenty women $(22 \%)$ used condoms for contraception.

At enrolment, participants reported on their sexual behaviour over the previous week. Women had a median of six sex acts (range 0-40) with five clients (range 0-40) (table 1). Nearly two thirds (64\%) said they had used condoms during their last sex act. More than one third (37\%) reported a history of genital ulcers. Twenty five participants $(27 \%)$ were rapid plasma reagin (RPR) seroreactive, and three $(3 \%)$ had both UTI symptoms and leucocytes in their urine.

The median diaphragm size dispensed at enrolment was $70 \mathrm{~mm}$, and the modal size was $65 \mathrm{~mm}$ (range $60-80 \mathrm{~mm}$ ); $95 \%$ of devices were between $65 \mathrm{~mm}$ and $75 \mathrm{~mm}$.

Trends in condom and diaphragm use are shown in table 2 and figure 1. At follow up, women answered questions about their sexual behaviour over the previous 2 days (compared with enrolment, where sexual behaviour was assessed over the previous week). Reported condom use remained roughly constant, increasing slightly from $61 \%$ of acts protected by condoms at enrolment, to $65 \%$ at the 1 week visit and $70 \%$ at the 4 week visit, decreasing slightly to $67 \%$ at the final visit (fig 1). Diaphragm use was also fairly constant: diaphragms were used in $97 \%$ of acts at 1 week, unchanged at 4 weeks, and $95 \%$ of acts at the 8 week visit. Condom failure (when a condom slipped off or ruptured) in the past week was common, reported by four women at the 1 week and 4 week follow up visits and 10 women at the final visit.

We also computed the absolute number (rather than proportion) of unprotected sex acts, where condoms or diaphragms were not used. The number of women reporting zero unprotected acts (in other words, that all acts were protected by condoms, either alone or together with the diaphragm), stayed approximately the same over time. At enrolment, 30 women (42\%) reported they used condoms at all sex acts; at both the 1 week and 4 week visits, 39\% of women reported they used condoms at all sex acts. At the final visit, 35 women (48\%) reported always using condoms. Conversely, the number of women reporting four or more sex acts without condoms decreased and then stabilised. Eighteen women $(25 \%)$ at enrolment reported four or more sex acts where condoms were not used, compared with seven participants $(9 \%)$ at the 1 week visit and five participants $(6 \%)$ at the 4 week visit. At the final visit, eight women (11\%) reported four or more sex acts without condoms.

We also assessed the absolute number of sex acts where women used diaphragms, either alone or together with condoms (table 2). In general, reported diaphragm use was high, with 67 women (89\%) at the 1 week visit, 67 women $(87 \%)$ at the 4 week visit, and 69 women (95\%) at the 8 week visit reporting they used their diaphragm at all sex acts. During the 8 week period, only one woman reported having sex four or more times without the diaphragm.

One third of participants $(n=31)$ reported problems involving 33 diaphragms during the study. Most were minor, such as discoloration or slight variations in shape. Eight diaphragms sustained major damage, including five when participants folded them at an inflexible part of the device rim. Twenty replacement diaphragms were dispensed, because five original devices were the wrong size, seven had been lost, and eight were seriously damaged.

Seven women became pregnant: five pregnancies were diagnosed at the 4 week visit and two additional pregnancies at the final visit. Because we tested for pregnancy at enrolment and not again until the 4 week visit, some women found to be pregnant at 4 weeks may have already been pregnant (though not clinically detectable) at enrolment. If all seven women truly became pregnant during their participation, the incidence rate is 53 pregnancies per 100 women years (exact 95\% confidence interval: 21 to 110 pregnancies per 100 woman years). No women requested EC during the study.

Participant reports of vaginal discharge and pelvic tenderness increased when diaphragms were initially dispensed, but returned to enrolment levels by the study's conclusion (table 3). Incident UTIs remained low at all visits and no women experienced repeat UTIs during the study. Clinical abnormalities on the vulva, vagina, cervix, uterus, and adnexes were also rare (maximum 9\% at any visit). No women were discontinued early because of protocol violations.

Table 2 Participants' reported sexual frequency, condom and diaphragm use by study visit*

\begin{tabular}{|c|c|c|c|c|c|c|c|c|}
\hline & \multicolumn{2}{|c|}{ Enrolment $(n=72) \dagger$} & \multicolumn{2}{|c|}{1 week visit $(n=75)$} & \multicolumn{2}{|c|}{4 week visit $(n=77)$} & \multicolumn{2}{|c|}{8 week visit $(n=73$ ) } \\
\hline & Median & (range) & Median & (range) & Median & (range) & Median & (range) \\
\hline Number of sex acts & 6 & $(0-40)$ & 3 & $(0-26)$ & 3 & $(0-26)$ & 3 & $(0-32)$ \\
\hline \multicolumn{9}{|c|}{ Women reporting sex acts where condoms were not used $\neq$} \\
\hline 0 acts & 30 & (42) & 29 & (39) & 30 & (39) & 35 & (48) \\
\hline $1-3$ acts & 24 & (33) & 39 & (52) & 42 & (55) & 30 & (41) \\
\hline 4 or more acts & 18 & (25) & 7 & (9) & 5 & (6) & 8 & (11) \\
\hline \multicolumn{9}{|c|}{ Women reporting sex acts where diaphragms were not used $\neq$} \\
\hline 0 acts & - & - & 67 & (89) & 67 & (87) & 69 & (95) \\
\hline $1-3$ acts & - & - & 8 & (11) & 10 & (13) & 3 & (4) \\
\hline 4 or more acts & - & - & 0 & (0) & 0 & (0) & 1 & (1) \\
\hline
\end{tabular}

*At enrolment women reported how many times they had sex and their use of condoms in the last week. At follow up visits, women reported how many times they had sex and their use of condoms and diaphragms in the last 2 days.

†At enrolment, tallies refer to sex acts protected by condoms alone.

fOnly among women reporting any sex acts. 
Table 3 Reproductive health and diaphragm use of participants during clinical examinations

\begin{tabular}{|c|c|c|c|c|c|c|c|c|c|c|c|}
\hline \multirow[b]{2}{*}{ Visit } & \multicolumn{2}{|c|}{$\begin{array}{l}\text { Vaginal } \\
\text { discharge/pelvic } \\
\text { tenderness }\end{array}$} & \multicolumn{2}{|c|}{$\begin{array}{l}\text { Any } \\
\text { abnormality* }\end{array}$} & Pregnant† & \multicolumn{2}{|c|}{ UTI } & \multicolumn{2}{|c|}{$\begin{array}{l}\text { Wore diaphragm } \\
\text { all day } \\
\text { yesterday? }\end{array}$} & \multicolumn{2}{|c|}{$\begin{array}{l}\text { Diaphragm } \\
\text { in place } \\
\text { during } \\
\text { examination }\end{array}$} \\
\hline & No & $\%$ & No & $\%$ & No $\%$ & No & $\%$ & No & $\%$ & No & $\%$ \\
\hline $\begin{array}{l}\text { Enrolment }(n=91) \\
1 \text { week }(n=91 \\
4 \text { week }(n=89) \\
8 \text { week }(n=87)\end{array}$ & $\begin{array}{r}7 \\
19 \\
16 \\
7\end{array}$ & $\begin{array}{r}8 \\
21 \\
18 \\
8\end{array}$ & $\begin{array}{l}2 \\
6 \\
6 \\
8\end{array}$ & $\begin{array}{l}2 \\
7 \\
7 \\
9\end{array}$ & $\begin{array}{lc}0 & 0 \\
\text { Not tested } \\
5 & 6 \\
6 & 7\end{array}$ & $\begin{array}{l}3 \\
3 \\
2 \\
0\end{array}$ & $\begin{array}{l}3 \\
3 \\
2 \\
0\end{array}$ & $\begin{array}{l}- \\
80 \\
82 \\
81\end{array}$ & $\begin{array}{l}- \\
88 \\
92 \\
93\end{array}$ & $\begin{array}{l}- \\
76 \\
82 \\
77\end{array}$ & $\begin{array}{l}- \\
84 \\
92 \\
89\end{array}$ \\
\hline
\end{tabular}

Continuous diaphragm use appeared highly acceptable: reported continuous use for the previous day increased from $87 \%$ at the 1 week visit to $93 \%$ at the 8 week visit. In addition, the number of women reporting diaphragm problems decreased from 15 participants $(16 \%)$ at the 1 week visit to six $(7 \%)$ at the final visit. Early in the study, participants' complaints included physical issues such as discomfort and awareness of the diaphragm by a partner during sex. Nearer the study end, problems focused on lost or damaged devices. Focus group discussions with these women also explored diaphragm acceptability, including women's opinions about use and whether they would recommend the diaphragm to friends (an analysis of these discussions is forthcoming).

Fifty one adverse events (including self reported events and those noted during clinical exams) were reported in 29 women. Nearly two thirds (65\%) were related to gynaecological symptoms such as vaginal discharge or itching $(n=14)$, genital ulcers $(\mathrm{n}=8$, three identified as probable herpes), dysuria $(n=3)$, proteinuria $(n=3)$, leucocyturia $(n=2)$, and cervicitis $(n=3)$; most others $(31 \%)$ related to gastrointestinal or influenza-like illness. No serious adverse events were reported.

\section{DISCUSSION}

Diaphragms were highly acceptable among these study participants. Our acceptability measures included the proportion of women reporting using diaphragms continuously during the previous day (ranged by follow up visit from $88 \%$ to $93 \%$ ), high retention (96\%), and low occurrence of product non-use. Problems decreased during the study, suggesting that women became more comfortable with their diaphragms over time and thus had fewer negative experiences.

We conclude that a trial of diaphragm use in this population is highly feasible. Possibly because of our work over several years with sex worker populations in Madagascar, we had a response rate of $100 \%$ and recruited our desired sample size quickly. In addition, our tracking and contact methods allowed us to follow $96 \%$ of participants successfully.

The study's primary limitation is the short duration of follow up. We are unable to generalise about diaphragm use over a long period of time based on these data; we will carefully measure patterns of diaphragm and condom use in the randomised trial. Nevertheless, we monitored changes in reported condom use to determine whether owning a diaphragm was associated with reduced condom use, since condom migration during the planned randomised trial would pose problems for both ethical and methodological reasons. We observed only minimal changes in condom use in this small sample. In addition, although condom use did not change substantially during follow up, the number of women reporting that all sex acts were protected by condoms was higher at the final visit than at any previous visit. We acknowledge, however, that social desirability bias cannot be ruled out. The results of focus group discussions may provide more insight about women's attitudes towards condom use.

It is possible that rates of diaphragm use, though quite high during this short follow up period, will eventually fall to those reported for condom use, since both are barrier methods. Nevertheless, we are hopeful that the other attributes of the diaphragm-that it is female controlled, and may be used covertly and continuously-may allow for higher usage rates over time.

We anticipated that participants' generally low level of schooling would necessitate extensive diaphragm education. For example, leaving the diaphragm in place for at least 6 hours after sex was difficult for women who lack watches. In other settings, new diaphragm users may be told that if they have sex before going to sleep, they should leave the device in place until they wake up the following morning. Because of sex workers' irregular hours, however, other strategies for estimating time since the last sex act are needed. Describing genital anatomy in simple terms was another challenge, and it was necessary to dispel common misconceptions about diaphragm use (for example, some women believed that when they urinated the device would fall out).

The pregnancy rate, 53 pregnancies per 100 women years, is substantially higher than the rates of $11-13$ pregnancies per 100 women years reported in trials evaluating the contraceptive efficacy of latex diaphragms plus spermicide. ${ }^{16}$ This finding is even more surprising considering that $24 \%$ of women reported using oral or injectable hormonal contraception at enrolment (including two of the women who eventually became pregnant). Women's non-use of EC may indicate that participants did not fully understand the diaphragm's limited contraceptive efficacy or their risk of pregnancy. Anecdotal evidence suggests that women are not familiar with EC in Madagascar, so even though clinicians discussed EC with participants, some may have remained hesitant about using it. In addition, EC was not free, which may have been a barrier to use. Other potential explanations for the high pregnancy rate include possible misuse of the diaphragm (such as not leaving it in for at least 6 hours after sex) and misreporting the frequency or consistency of use of diaphragms, condoms, or other contraceptive methods. Finally, sex workers' attitudes towards pregnancy are not well understood, and some pregnancies may have been desired if not planned. Pregnancy prevention in the randomised trial will be addressed in greater detail in light of these findings, and we will offer free hormonal contraception to all participants.

An unexpected number of diaphragms were damaged during the short follow up period. This particular diaphragm 


\section{Key messages}

- In regions where incidence of sexually transmitted infections (STI) is high and women lack the social and economic power to insist that their partners remain monogamous or use condoms, new "women controlled" methods for disease prevention are very much needed. The diaphragm, a reusable contraceptive device approved worldwide for prevention of pregnancy, may be an effective option for control of some STIs

- Continuous diaphragm use, without lubricant or spermicide, was acceptable and feasible in a resource poor, low education sex worker population

- According to self reports, women's use of condoms did not decline when they began using diaphragms; in addition, diaphragm use was very high throughout follow up (diaphragms used in $95 \%-97 \%$ of sex acts)

product, the Wide-Seal Arcing Diaphragm, has arrows marked on the rim to indicate the appropriate place to fold the device before insertion. Milex Products conducted an analysis of all devices after the study to determine whether the damage was the result of improper use or a manufacturing error; they concluded that much of the damage occurred in devices which became deformed when participants forced them to fold at an inflexible place. To avoid similar damage in the randomised study, we will use a diaphragm with an "Omniflex" design, which may be folded in any place along the rim.

This study reinforces the need for woman controlled methods of disease prevention. During the 8 week period, 17 participants, or almost one in five women, reported that a condom had slipped off or ruptured at least once within the past week. Thus, although many sex workers reported using condoms, this method is far from failsafe. The availability of an additional method of STI prevention may prove highly valuable to millions of vulnerable women worldwide.

\section{ACKNOWLEDGEMENTS}

This project was supported by the National Institute of Allergy and Infectious Diseases (Sexually Transmitted Diseases Clinical Trials Unit, NOl-AI-75329), and additional support was received from the United States Agency for International Development in Antananarivo. The views and opinions presented do not reflect those of the funding agencies. We thank Andry Rasamindrakotroka, Wendy Githens Benazerga, Jocelyne Andriamiadana, Myron Cohen, and Barbara Savarese for their support.

\section{CONTRIBUTORS}

FB conceived and designed the study, provided guidance throughout its implementation, and supervised the analysis and writing processes; ANT conducted the analysis and wrote the manuscript;
KVD assisted with the questionnaire design, supervised the field work and provided extensive comments during manuscript development; NLR and NR assisted with the questionnaire design, collected and cleaned the data, and edited the manuscript; KZ contributed to the study protocol, provided medical training to staff, and edited the manuscript; JRR provided scientific and institutional support, assisted with protocol and questionnaire development, and provided feedback on early drafts of the manuscript.

\section{Authors' affiliations}

F Behets, A N Turner, Department of Epidemiology, University of North Carolina at Chapel Hill, Chapel Hill, NC, USA

F Behets, K Van Damme, Department of Medicine, University of North Carolina at Chapel Hill, Chapel Hill, NC, USA

K Van Damme, N L Rabenja, N Ravelomanana, UNC-MAD,

Antananarivo, Madagascar

K Zeller, William J Clinton Presidential Foundation, New York, NY, USA

J R Rasolofomanana, Institut National De Santé Publique et

Communautaire, Ecole de Médecine, Antananarivo, Madagascar

Conflict of interest: No authors have any conflict of interest.

\section{REFERENCES}

1 Stein ZA. HIV prevention: the need for methods women can use. Am J Public Health 1990;80:460-2.

2 Kennedy DM. Birth control in America: the career of Margaret Sanger. New Haven: Yale University Press, 1970.

3 Moench TR, Chipato T, Padian NS. Preventing disease by protecting the cervix: the unexplored promise of internal vaginal barrier devices. AIDS 2001;15:1595-602.

4 Bird ST, Harvey SM, Maher JE, et al. Acceptability of an existing, femalecontrolled contraceptive method that could potentially protect against HIV: a comparison of diaphragm users and other method users. Womens Health Issues 2004;14:85-93.

5 van der Straten A, Kang MS, Posner SF, et al. Predictors of diaphragm use as a potential sexually transmitted disease/HIV prevention method in Zimbabwe. Sex Transm Dis 2005;32:64-71.

6 Rosenberg MJ, Davidson AJ, Chen JH, et al. Barrier contraceptives and sexually transmitted diseases in women: a comparison of female-dependent methods and condoms. Am J Public Health 1992;82:669-74.

7 Austin H, Louv WC, Alexander WJ. A case-control study of spermicides and gonorrhea. JAMA 1984;251:2822-4.

8 Magder LS, Harrison HR, Ehret JM, et al. Factors related to genital Chlamydia trachomatis and its diagnosis by culture in a sexually transmitted disease clinic. Am J Epidemiol 1988;128:298-308.

9 Kelaghan J, Rubin GL, Ory HW, et al. Barrier-method contraceptives and pelvic inflammatory disease. JAMA 1982;248:184-7.

10 Wolner-Hanssen P, Eschenbach DA, Paavonen J, et al. Decreased risk of symptomatic chlamydial pelvic inflammatory disease associated with oral contraceptive use. JAMA 1990;263:54-9.

11 Behets F, Andriamiadana J, Rasamilalao D, et al. Sexually transmitted infections and associated socio-demographic and behavioural factors in women seeking primary care suggest Madagascar's vulnerability to rapid HIV spread. Trop Med Int Health 2001;6:202-11.

12 Behets FM, Rasolofomanana JR, Van Damme K, et al. Evidence-based treatment guidelines for sexually transmitted infections developed with and for female sex workers. Trop Med Int Health 2003;8:251-8.

13 Fleming DT, Wasserheit JN. From epidemiological synergy to public health policy and practice: the contribution of other sexually transmitted diseases to sexual transmission of HIV infection. Sex Transm Infect 1999;75:3-17.

14 Cohen MS. Sexually transmitted diseases enhance HIV transmission: no longer a hypothesis. Lancet 1998;351(Suppl 3):5-7.

15 Corbett EL, Steketee RW, ter Kuile FO, et al. HIV-1/AIDS and the control of other infectious diseases in Africa. Lancet 2002;359:2177-87.

16 Kuyoh MA, Toroitich-Ruto C, Grimes DA, et al. Sponge versus diaphragm for contraception: a Cochrane review. Contraception 2003;67:15-18. 